Vaccine Volume 24, Issues 44-46, 10 November 2006, Pages 6717-6723

Proceedings of the Second European Influenza Conference, Proceedings of the

Second European Influenza Conference

doi:10.1016/j.vaccine.2006.05.068

Copyright (c) 2006 Elsevier Ltd All rights reserved.

\title{
Programme of the Community Network of Reference Laboratories for Human Influenza to Improve Influenza Surveillance in Europe
}

\section{Adam Meijer ${ }^{a}, \mathrm{~b}, *$, Caroline Brown ${ }^{\mathrm{a}}, 1$, Olav Hungnes ${ }^{\mathrm{c}}$, Brunhilde Schweiger ${ }^{d}$, Martine Valette ${ }^{e}$, Sylvie van der Werf ${ }^{f}$, Maria Zambon ${ }^{g}$ and on behalf of the members of the Virology Task Groups of the European Influenza Surveillance Scheme ${ }^{2}$}

${ }^{a}$ Netherlands Institute for Health Services Research (NIVEL), European Influenza Surveillance Scheme Co-ordination Centre, Otterstraat 118-124, 3513 Utrecht, $\mathrm{CR}$, The Netherlands

${ }^{\mathrm{b}}$ National Institute of Public Health and the Environment, Diagnostic Laboratory for Infectious Diseases and Screening, Bilthoven, The Netherlands

${ }^{\mathrm{c}}$ National Influenza Centre (NIC), Norwegian Institute of Public Health, Oslo, Norway

${ }^{d}$ NIC, Robert Koch Institute, Berlin, Germany

eNIC Région-Sud, Hospices Civils de Lyon, Laboratoire de Virologie, Lyon, France

${ }^{f}$ NIC Région-Nord, Institut Pasteur, Paris, France

${ }^{9}$ NIC, Respiratory Virus Unit, ERNVL, Health Protection Agency, London, United Kingdom

* Corresponding author. Tel.: +31 30 2729857; fax: +31 302729729.

${ }^{1}$ Current address: WHO Regional Office for Europe, Copenhagen, Denmark.

${ }^{2}$ Aubin J-T (France), Blaskovicova H (Slovakia), Brown C (EISS-CC, The Netherlands), Brytting M (Sweden), Collins T (Scotland, UK), Coughlan S (Ireland), Havlíckové M (Czech Republic), Hay A (England), Hungnes O (Norway), de Jong J (The Netherlands), Machala M (Poland), Kalnina V-I (Latvia), Lina B (France), Meijer A (EISS-CC, The Netherlands), Nielsen LP (Denmark), PérezBreña P (Spain), Pregliasco F (Italy), Rebelo de Andrade H (Portugal), Rimmelzwaan G (The Netherlands), Schweiger B (Germany), Thomas $Y$ (Switzerland), Valette M (France), van der Werf S (France), Wiman $\AA$ (Sweden), Zambon M (England, UK), Ziegler T (Finland).

\begin{abstract}
All laboratories participating in the Community Network of Reference Laboratories for Human Influenza in Europe (CNRL) co-ordinated by the European Influenza Surveillance Scheme (EISS) should be able to perform a range of influenza diagnostics. This includes direct detection, culture, typing, subtyping and strain characterisation of influenza viruses, diagnostic serology and the creation of archives for clinical specimens and virus isolates. To improve the capacity and quality of the laboratories of the CNRL and to increase the consistency in performance among all 25 European Union countries plus Norway, Romania, and Switzerland, five task groups were set up in February 2005. These task groups
\end{abstract}


Vaccine Volume 24, Issues 44-46, 10 November 2006, Pages 6717-6723

Proceedings of the Second European Influenza Conference, Proceedings of the Second European Influenza Conference

developed work programmes in the areas of virus isolation, antibodies, molecular virology, quality control assessment and antiviral susceptibility testing. This report outlines the programmes and the results achieved in the first half-year of operation of the task groups. The action plans are challenging and it is expected that these efforts will lead to considerable improvements in the performance of the laboratories and in the standardisation of methods employed in Europe with regard to routine influenza surveillance and early warning for emerging viruses.

Keywords: Influenza; Laboratory network; European Influenza Surveillance Scheme

\section{Introduction}

The European Influenza Surveillance Scheme (EISS) has evolved from its start in 1996 [1] into an organisation that includes A D. 2005 all 25 European Union member states plus Norway, Romania and Switzerland. The main objective of EISS is to help reduce the burden of disease associated with influenza in Europe by collecting and exchanging timely information on influenza activity and circulating influenza viruses. As part of the evolution of EISS, the Community Network of Reference Laboratories for Human Influenza in Europe (CNRL) was established in 2003 [2]. As of October 2005, the CNRL comprises 38 laboratories of which at least one is located in each of the 28 countries in EISS. All WHO National Influenza Centres in the 28 countries are included in the CNRL.

The laboratories in the CNRL should be able to perform a range of influenza diagnostics defined as basic tasks [2]. These include direct detection, culture, typing, subtyping and strain characterisation of influenza viruses, diagnostic serology and the creation of archives for clinical specimens and virus isolates. Influenza surveillance data is published by EISS on its website (www.eiss.org) and in the EISS weekly electronic bulletin (http://www.eiss.org/cgi-

files/bulletin_v2.cgi) and integrates virological information produced by the laboratories with information on the incidence of influenza-like-illness and/or acute respiratory infection (see Fig. 1 for example graphs). The performance of the 32 laboratories included in the CNRL at the end of 2004 was evaluated by inventories and quality control studies and these indicated that improvements were needed in certain areas of virus testing [2], [3], [4] and [5]. Task groups were therefore established with the aim to improve the capacity and quality of the laboratories of the CNRL and to achieve consistent performance in routine influenza surveillance and detection of emerging viruses among all 28 countries included in EISS. 
Vaccine Volume 24, Issues 44-46, 10 November 2006, Pages 6717-6723

Proceedings of the Second European Influenza Conference, Proceedings of the Second European Influenza Conference

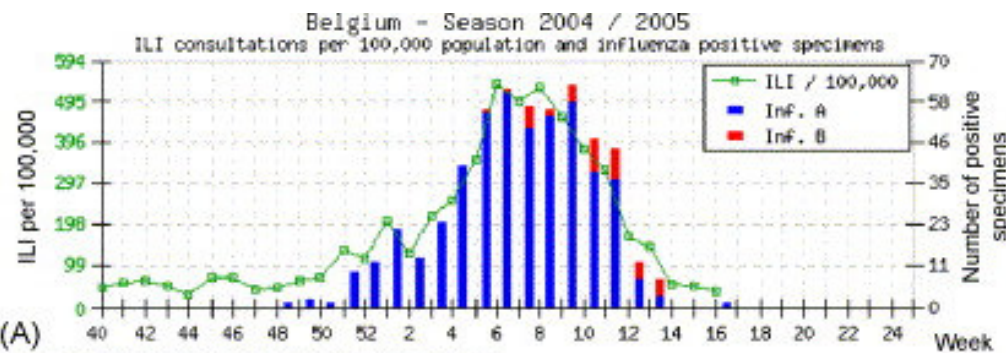

A) Source: Europeen Influenca surveillance schewe Complied at 12.59 on Apc 282005

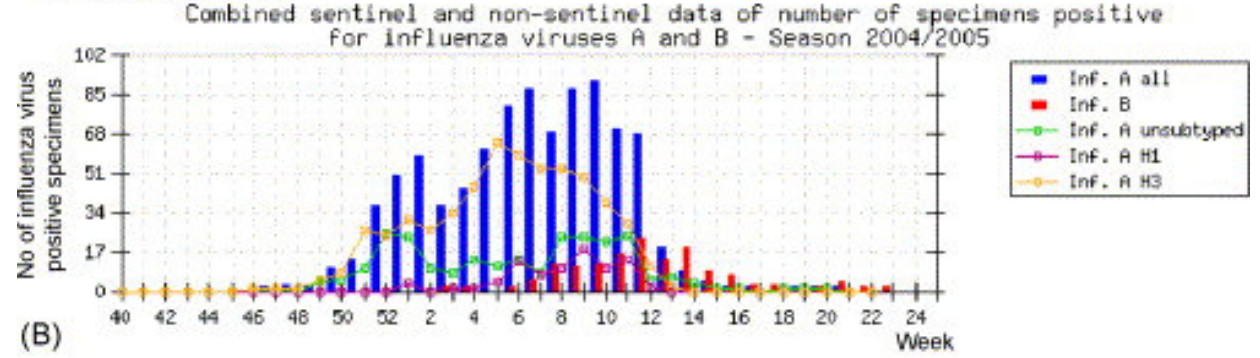

Created in 00:18 on oet 282005

Source: Eurcpean Influenzs Surveillance Schere

Cumulative influenza virus isolate typings and subtypings* Season $2004 / 2005$

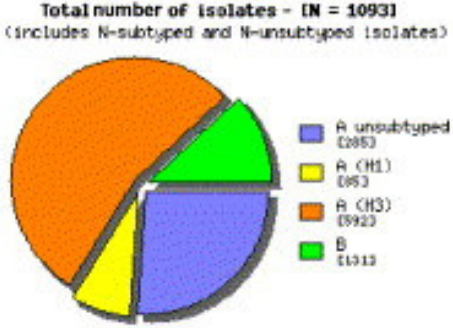

Number of isolates $\mathrm{N}$-subtyped $-[\mathrm{H}=402]$

(C)

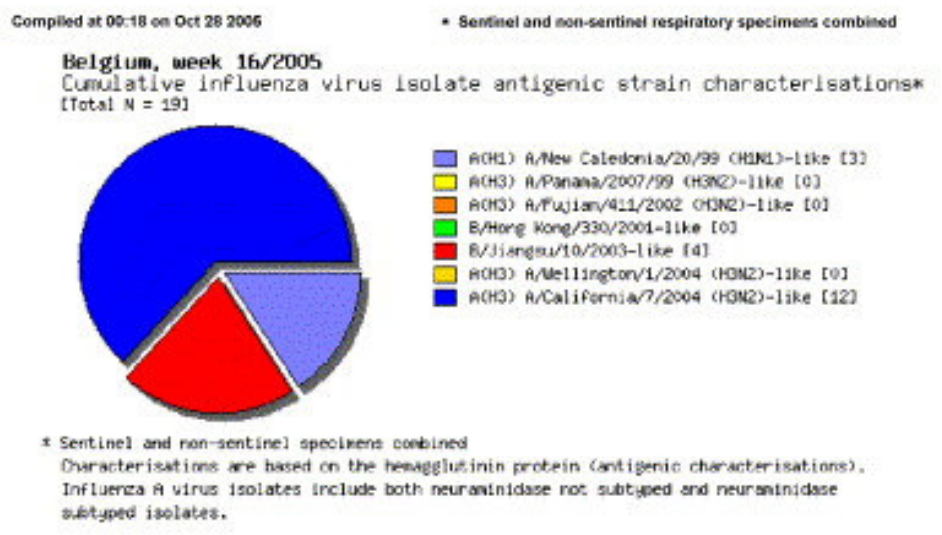

(D)

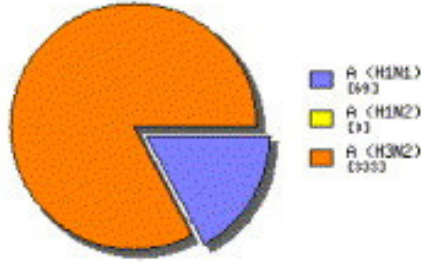
shetyped isolates.

Fig. 1. Examples of graphs published by EISS. The graphs are from Belgium for the season 2004/2005: (A) combined graph of weekly sentinel data; influenzalike-illness consultations per 100,000 population and number of influenza positive specimens; (B) combined graph of weekly sentinel and non-sentinel virus detections; (C) Pie chart of cumulative number of typed and subtyped viruses for the whole season; and (D) Pie chart of cumulative number of antigenically characterised viruses for the whole season. 
Vaccine Volume 24, Issues 44-46, 10 November 2006, Pages 6717-6723

Proceedings of the Second European Influenza Conference, Proceedings of the Second European Influenza Conference

\section{Methods}

During the Ninth EISS Annual Meeting in May 2004 in Sintra, Portugal the EISS virologists decided to start five task groups in the areas of virus isolation, antibodies, molecular virology, quality control assessment, and antiviral susceptibility testing. Invitations to participate and, where appropriate, to contribute by carrying out work in the laboratory were sent to the EISS virologists in autumn 2004. Twenty-five expert virologists from 17 European countries signed up for a task group (see footnote 2). To each task group a member of the EISS co-ordination centre was appointed to assist the task group. Draft work programmes were made and were discussed and finalized during a meeting of all participants of the five task groups in February 2005 in Noordwijkerhout, The Netherlands. The written finalised work programmes were communicated to the EISS members by email and discussed at the Tenth EISS Annual Meeting in May 2005 in Birmingham, UK. To spread the workload of the task group members it was agreed that, where needed, additional EISS experts would be asked to help in carrying out the laboratory work. Every half year each task group writes a progress report, which will be distributed among the EISS members. This paper outlines the programmes and the results achieved in the first half-year of operation of the task groups.

\section{Results}

\subsection{Virus isolation}

The objectives of the virus isolation task group are: (i) to develop methods for highly sensitive standardised virus isolation; (ii) to secure the availability of egg isolated viruses as vaccine candidates; and (iii) to develop a protocol for biosafety in relation to isolation of viruses of different pathogenicity.

To achieve highly sensitive standardised virus isolation, different cell-lines will be compared using archived clinical specimens with known viral content based on previous culture attempts and nucleic acid amplification techniques. Based on a literature study and experiences of the task group members, the following candidate cell lines have been selected: MDCK-II (ATCC CCL-34); MDCK-I; serum free MDCK; MDCK clone CB4; MDCK-Siat cells; LLC-MK2; and HepG2 cells [6], [7], [8], [9], [10] and [11]. The clinical specimens will comprise a selection of specimens positive for influenza $A(H 3 N 2), A(H 1 N 1), A(H 1 N 2)$, B Yamagata lineage, and $B$ Victoria lineage viruses and a selection of negative specimens, from different seasons. The cell lines will also be tested for susceptibility to avian viruses infecting humans, $A(H 5)$ and $A(H 7)$ viruses having the highest priority. In addition, the cell lines will be tested for their suitability for virus titration and neutralisation assays. Of the optimal cell line stocks will be grown and a distribution mechanism will be set up to secure the availability for the entire network. A standardized protocol for virus isolation will be developed taking into consideration the type of culture (shell-vial, tube culture, rolling drum), inoculum (nature of specimen, volume, transport medium, centrifugation, etc.), time and temperature of adsorption and removal of inoculum, medium (composition, $\mathrm{pH}$ control, serum, $\mathrm{CO}_{2}$, etc.), use of trypsin (source and concentration), temperature and duration of incubation, and the assessment of growth (ELISA, hemagglutination $[\mathrm{HA}]$, cytopathic effect $[\mathrm{CPE}]$, immuno-fluorescence assay [IFA]).

Egg-isolated viruses required for selection of vaccine candidates are not readily available as most laboratories in the network have moved from eggs to cell lines 
Vaccine Volume 24, Issues 44-46, 10 November 2006, Pages 6717-6723

Proceedings of the Second European Influenza Conference, Proceedings of the Second European Influenza Conference

for virus isolation and growth. The task group will define criteria for the selection of representative viruses of a given season for egg isolation within EISS, such as: minimum number of egg isolates per season for each type and subtype ( $A(H 3 N 2)$, $A(H 1 N 1), A(H 1 N 2), B$ Yamagata lineage, and B Victoria lineage viruses) according to characteristics of antigenic variants. In addition, mechanisms will be established to: (i) share the workload among laboratories within EISS that are able to perform primary egg isolation; (ii) share information about availability of primary egg isolates within EISS; and (iii) to identify the needs for egg isolation of emerging antigenic variants (in concert with the WHO collaboration centre [WHO-CC] in London, UK).

Isolation from routine specimens is carried out under bio-safety level (BSL) 2 conditions whereas isolation from specimens from avian flu suspect cases should be carried out under BSL3+ conditions [12]. In a recent inventory, 25 of 31 laboratories in EISS reported having access to a BSL3 facility [2]. The task group will develop a general procedure for stepping up the bio-safety level for performing virus isolation from specimens from suspect cases of highly pathogenic/new subtype viruses.

\subsection{Antibodies}

The goal of the antibodies task group is to improve and harmonize methods for identification and characterization of influenza viruses and influenza virus infections since these procedures are fundamental tools in influenza surveillance, epidemiological studies as well as in the evaluation of vaccine immunogenicity. The following work programme has been defined: (i) selection of most appropriate red blood cells for hemagglutination inhibition test (HI); (ii) standardization of antibodies for ELISA and IFA; (iii) securing the availability of up to date ferret sera and reference viruses for antigenic characterisation of circulating and emerging viruses; (iv) defining an algorithm for reporting of the dominant (sub)type; and ( $v$ ) standardisation of methods used for influenza serology.

To select the most appropriate red blood cells first the available information in the literature and from personal investigation has been reviewed. Four types of red blood cells, from chicken, guinea pig, turkey and human origin, are widely used. Because the performance of these red blood cells in HA and HI depends on type, subtype and year of isolation of human influenza viruses, all four types of red blood cells will be included in prospective studies for HA and HI with currently circulating human influenza viruses. As horse red blood cells are a better substrate for HA and HI of avian influenza viruses [13] and [14] these will be included for evaluation of $\mathrm{HA}$ and $\mathrm{HI}$ with $\mathrm{H} 5, \mathrm{H} 7$ and $\mathrm{H} 9$ virus strains with the highest priority. Based on the results a standard operating procedure will be written.

As several laboratories in the CNRL use ELISA or IFA for antigen detection in clinical specimens, the most critical factor in these tests, i.e. the antibody which binds to the antigen, will be evaluated by comparison with culture and nucleic acid amplification techniques (NAT) to select the optimal antibody/ies and method(s). Previously, commercially available near patient tests for rapid antigen detection have been evaluated by EISS [15].

To enhance the ability of all laboratories to characterise isolated viruses antigenically in a standard way, reference ferret antisera and viruses are distributed by the WHO-CC, London. To enhance preparedness for detection of 
Vaccine Volume 24, Issues 44-46, 10 November 2006, Pages 6717-6723

Proceedings of the Second European Influenza Conference, Proceedings of the Second European Influenza Conference

possible pandemic viruses, arrangements will be made with the WHO-CCs and the Community Reference Laboratory for Avian Influenza, Weybridge, UK to supply reference reagents for antigenic characterisation of $\mathrm{H} 2, \mathrm{H} 5, \mathrm{H} 7$ and $\mathrm{H} 9$ subtype influenza A viruses.

Algorithms for the reporting of the dominant circulating type or subtype indicator, including co-dominance of two types or subtypes, for a country (Fig. 2) and for Europe (not shown) as a whole have been developed and application will start as of the 2005/2006 season. This will greatly improve the comparability of the indicator between the European countries.

1. If possible, the dominant type/subtype should be reported when 10 or more positive results are available. In small countries or regions (e.g. countries/regions with a population of less than 5 million), the assessment can be based on a smaller number of positive results.

2. If possible, the dominant type/subtype should be based on results available for the previous week. However, 1) at the beginning and end of the season, 2) in small countries/regions and 3) in countries where there are not so many samples, the assessment can be based on the previous TWO weeks.

3. Definition of a co-dominant type

The threshold for co-dominance is set at $40 \% / 60 \%$.

Examples:

$60 \% \mathrm{~A}$ and $40 \% \mathrm{~B}: \mathrm{A}+\mathrm{B}$

$55 \% \mathrm{~A}$ and $45 \% \mathrm{~B}: \mathrm{A}+\mathrm{B}$

$50 \% \mathrm{~A}$ and $50 \% \mathrm{~B}: \mathrm{A}+\mathrm{B}$

but $65 \% \mathrm{~A}$ and $35 \% \mathrm{~B}$; only $\mathrm{A}$

$61 \% \mathrm{~A}$ and $39 \% \mathrm{~B}$ : only $\mathrm{A}$

4. If you want to report the $\mathrm{H}$ (and $\mathrm{N}$ ) subtype of influenza $\mathrm{A}$ viruses in the dominant (sub)type, at least $50 \%$ of influenza A viruses should have been subtyped

5. Definition of a co-dominant influenza A subtype

The threshold for co-dominance is set at $40 \% / 60 \%$

If possible, there should be 10 influenza A subtypes to perform this analysis (however, points 1 and 2 above apply)

Examples:

$60 \% \mathrm{~A}(\mathrm{H} 3 \mathrm{~N} 2)$ and $40 \% \mathrm{~A}(\mathrm{H} 1 \mathrm{~N} 1): \mathrm{H} 3 \mathrm{~N} 2+\mathrm{H} 1 \mathrm{~N} 1$

$55 \% \mathrm{H}_{3}$ and $45 \% \mathrm{H}_{1}: \mathrm{H}_{3}+\mathrm{H}_{1}$

$50 \% \mathrm{H} 3$ and $50 \% \mathrm{~B}: \mathrm{H} 3+\mathrm{B}$

$70 \% \mathrm{~A}(\mathrm{H} 3 \mathrm{~N} 2): \mathrm{H} 3 \mathrm{~N} 2$

but $65 \% \mathrm{H} 3 \mathrm{~N} 2$ and $35 \% \mathrm{~B}$ : only $\mathrm{H} 3 \mathrm{~N} 2$ $61 \% \mathrm{H} 3$ and $39 \% \mathrm{H} 1$ : only $\mathrm{H} 3$

6. These are general rules and the national expert should take into consideration a number of factors (local outbreaks, holidays, geographical differences) when judging week-to-week developments. The national expert should apply these rules with an appreciation of special conditions, local knowledge and expertise.

Fig. 2. Recommended algorithm to retrieve the weekly dominant type and subtype indicator.

The main methods applied for serology are $\mathrm{HI}$ and virus neutralisation, and these are well established for serology after infection by or vaccination with human 
Vaccine Volume 24, Issues 44-46, 10 November 2006, Pages 6717-6723

Proceedings of the Second European Influenza Conference, Proceedings of the Second European Influenza Conference

influenza viruses. However, selection of the best performing red blood cells in HI and cell lines in virus neutralisation assays by the virus isolation task group will further harmonize the serological assays. In addition, the antibodies task group will take into consideration the special needs for serology after infection with avian influenza virus in their recommendations [13], [14] and [16].

\subsection{Molecular virology}

The objectives of the molecular virology task group are: (i) to secure the availability of molecular control reagents and creating a distribution system for these and (ii) to secure access to molecular sequence data.

In view of the current situation with $\mathrm{A}(\mathrm{H} 5 \mathrm{~N} 1)$ in Asia and Europe, creation and distribution of controls for this virus has top priority. Plasmid based $\mathrm{H} 5$ controls and run-off H5 RNA transcripts have been constructed and made available to the CNRL laboratories. The main problem noted here is keeping up-to-date with the drift in the $\mathrm{H}$ sequence of $\mathrm{A}(\mathrm{H} 5 \mathrm{~N} 1)$ viruses. Further controls in addition to $\mathrm{H} 5$ will be constructed with the following priority: (i) $\mathrm{H} 7$ avian influenza; (ii) $\mathrm{H} 2$ (the only non-circulating human subtype with documented pandemic potential); (iii) $\mathrm{H}$ of other avian influenza viruses with zoonotic cases, i.e. $\mathrm{H} 9$ and $\mathrm{H} 10$; (iv) $\mathrm{H}$ of currently circulating human influenza viruses; and ( $v$ ) other genes for typespecific detection. It is a goal of the task group that the availability of controls shall not be a limiting resource for development and utilisation of molecular diagnostics for these viruses. For neuraminidase a need is envisaged to have suitable control materials to verify the ability of methods to detect and identify N1 genes of non-human origin, especially of the current $A(H 5 N 1)$ outbreak strains. The controls will preferably be constructed in a way that they can be easily differentiated from wild type sequences in case of a suspicion of contamination. Newly developed and existing molecular controls will, where possible, be banked at a central location in Europe at one of the CNRL laboratories and distributed from there.

In order to be able to rapidly share sequences of viruses isolated early in and during the season, deviating viruses, emerging viruses, PCR primers and probes, and molecular controls, a centralised influenza sequence database specifically designed for the needs of the CNRL has been set up at the Influenza Sequence Database (ISD) in Los Alamos, USA with a mirror site in Paris, France to secure continuous access (Fig. 3). This facility will further enhance the early warning function of the CNRL. In addition, it offers extensive possibilities to share sequences between individuals and with other important players in the field of influenza, e.g. the WHO-CCs and the European Vigilance against Viral Resistance (VIRGIL) and global Neuraminidase Inhibitor Susceptibility Network (NISN) projects on antiviral resistance. 
Vaccine Volume 24, Issues 44-46, 10 November 2006, Pages 6717-6723

Proceedings of the Second European Influenza Conference, Proceedings of the Second European Influenza Conference

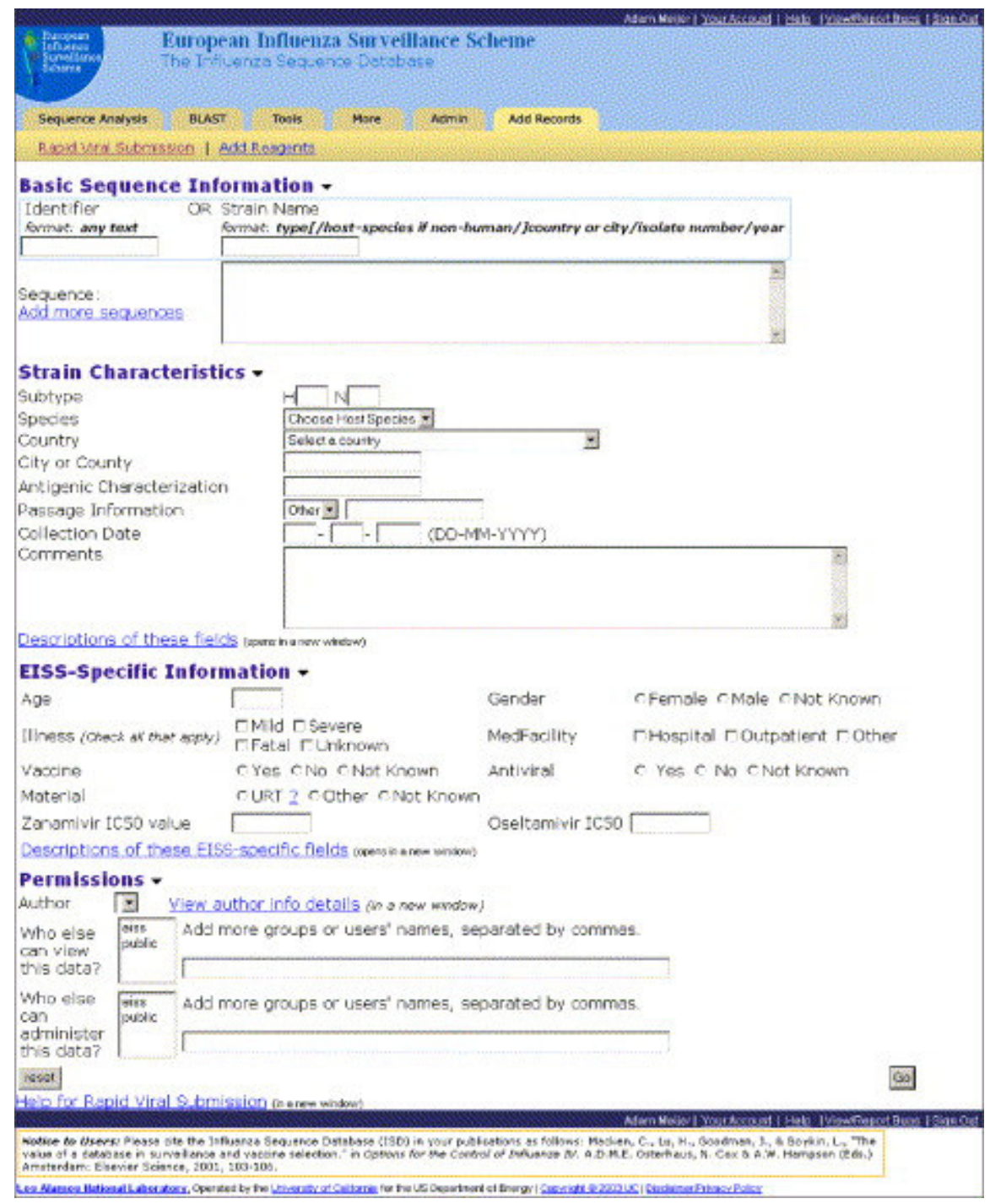

Fig. 3. Entry screen of the EISS private compartment at the Influenza Sequence Database in Los Alamos, USA, showing the EISS specific fields and the extensive possibilities for sharing of molecular sequence data.

\subsection{Quality control assessment}

The objectives of the Quality Control Assessment (QCA) task group are: (i) to organise QCAs; (ii) to prepare and distribute quality control panels; (iii) to analyse and report the results; and (iv) to organise follow-up help.

To assess the performance of the laboratories in the CNRL basic tasks [2], regular QCAs studies will be carried out for virus culture (including typing, subtyping and strain characterisation), molecular detection and serology. Follow-up activities of the QCA will include organised help for laboratories that do not pass the pre-set QCA criteria using the expertise of the other task groups. Virus culture QCA has been conducted in 2000 and was repeated in 2002 [2] and [3]. The improvement in performance between the two QCAs was considerable. The next virus culture QCA will be carried out in Autumn 2005. For molecular detection, connection with existing programs will be sought (www.qcmd.org). Because of the high bio-safety 
Vaccine Volume 24, Issues 44-46, 10 November 2006, Pages 6717-6723

Proceedings of the Second European Influenza Conference, Proceedings of the Second European Influenza Conference

requirements and costs for preparation of an $\mathrm{H} 5$ panel, this program does not include the $\mathrm{A}(\mathrm{H} 5 \mathrm{~N} 1)$ virus. Additional funding for molecular QCA including an $\mathrm{H} 5$ panel and serology will be sought.

\subsection{Antiviral susceptibility}

The objective of the antiviral susceptibility task group is to implement standardised antiviral susceptibility testing for EISS.

The EISS members intend to extend the influenza surveillance activities in Europe to include antiviral susceptibility since antiviral use is becoming more common for treatment and prevention where seasonal vaccination fails. So far, the number of naturally resistant viruses that have been identified worldwide has been very low, but development of resistance in humans has been reported [17] and [18]. To obtain an insight into the use of antivirals, EISS will collect aggregated data on prescriptions of antivirals (number of prescriptions for ILI cases per week or ARI cases per week). To monitor whether antiviral resistant strains emerge, EISS will collect aggregated data on the antiviral susceptibility of influenza virus isolates. In order to link susceptibility with antiviral use, EISS members have been recommended to include questions on antiviral use on the form that comes with the clinical specimen. To distinguish between resistant strains developing in a patient and transmission of resistant strains, the questions include the use of antivirals by the patient and by household contacts. To obtain the right clinical specimen for antiviral susceptibility analysis, the task group will also work on the standardisation of a swabbing protocol, as big differences exist between countries in swabbing techniques (throat/nose and/or combination). Information on nonresistant strains is also needed to determine background susceptibility and seasonal drift of susceptibility. The EISS private compartment for influenza molecular sequences in the ISD will contain data on antiviral susceptibility (Fig. 3 ), which will be linked to the virological surveillance and clinical incidence graphs at the EISS website and to data in the VIRGIL database. All these activities will be carried out in close collaboration with the VIRGIL project that has partly a similar aim. VIRGIL will develop a standard operating procedure for antiviral susceptibility testing which then each laboratory can implement. A training for using the method has also been foreseen in the VIRGIL project. For QC purposes VIRGIL will compose a panel of resistant viruses that will be available on request to CNRL members.

\section{Conclusion}

The task groups have proven to be useful forums for exchange of knowledge and expertise and starting up and realising collaborative projects to improve and standardise the laboratory work related to influenza surveillance in Europe. Concrete achievements in the short period of the existence of the task groups are: (i) distribution by the WHO-CC, London, UK of references ferret sera and viruses to all laboratories in the CNRL; (ii) recommendations for reporting of the dominant subtype; (iii) construction and distribution of $\mathrm{H} 5$ molecular controls; (iv) establishment of an EISS private compartment at the Influenza Sequence Database, Los Alamos, USA; (v) organisation of the third QCA for virus culture; and $(\mathrm{vi})$ recommendations for a standardized clinical specimen form.

The continued implementation of the action plans will further improve the quality of the performance of the laboratories in the CNRL and contribute to the standardisation of the methods used across Europe. This will lead to improved influenza surveillance and early warning for emerging viruses in Europe, which is 
Vaccine Volume 24, Issues 44-46, 10 November 2006, Pages 6717-6723

Proceedings of the Second European Influenza Conference, Proceedings of the Second European Influenza Conference

of particular importance with regard to the current thread of the spread of avian influenza $A(H 5 N 1)$ and transmission of this virus to humans.

\section{Acknowledgements}

EISS is partly funded by the Health and Consumer Protection Directorate-General of the European Commission. We thank all participating sentinel physicians, epidemiological institutes and laboratories across Europe for their ongoing support and contribution to EISS and the CNRL. We thank Paul Taylor, Quad Logic, Paris, France for developing and maintaining the EISS databases and website and for housing the European mirror of the ISD, Catherine Macken and Rachel Richard, ISD, Los Alamos, USA for developing and maintaining the EISS private compartment at the ISD, Liesbeth Meuwissen, EISS co-ordination centre, for critical reading of the manuscript and valuable comments, and John Paget, EISS co-ordination centre, for editing the manuscript.

\section{References}

[1] D.M. Fleming, J. van der Velden and W.J. Paget, The evolution of influenza surveillance in Europe and prospects for the next 10 years, Vaccine 21 (2003), pp. 1749-1753.

[2] A. Meijer, M. Valette, J.C. Manuguerra, P. Perez-Brena, J. Paget and C. Brown et al., Implementation of the community network of reference laboratories for human influenza in Europe, $J$ Clin Virol 34 (2005), pp. 87-96.

[3] M. Valette and M. Aymard, Quality control assessment of influenza and RSV testing in Europe: 2000-2001 season, Euro Surveill 7 (2002), pp. 161-165.

[4] T.J. Meerhoff, W.J. Paget, J.F. Aguilera and J. van der Velden, Harmonising the virological surveillance of influenza in Europe: results of an 18-country survey, Virus Res 103 (2004), pp. 31-33.

[5] T.J. Meerhoff, A. Meijer and W.J. Paget, EISS. Methods for sentinel virological surveillance of influenza in Europe - an 18-country survey, Euro Surveill 9 (2004), pp. 34-38.

[6] A.L. Frank, R.B. Couch, C.A. Griffis and B.D. Baxter, Comparison of different tissue cultures for isolation and quantitation of influenza and parainfluenza viruses, J Clin Microbiol 10 (1979), pp. 3236.

[7] M. Matrosovich, T. Matrosovich, J. Carr, N.A. Roberts and H.D. Klenk, Overexpression of the alpha2,6-sialyltransferase in MDCK cells increases influenza virus sensitivity to neuraminidase inhibitors, $J$ Virol 77 (2003), pp. 8418-8425.

[8] H. Meguro, J.D. Bryant, A.E. Torrence and P.F. Wright, Canine kidney cell line for isolation of respiratory viruses, J Clin Microbiol 9 (1979), pp. 175-179.

[9] Meijer A, van de Kamp EEHM, Koch G, Kimman TG. Cell-ELISA for antiviral susceptibility testing of influenza virus: performance depends on the compatibility of virus strain and type of MDCK cells.

Proceedings of the International Conference on Options of the Control of Influenza V, Okinawa, Japan 7-11 October 2003. International Congress Series 2004; 1263:65-8.

[10] L. Ollier, A. Caramella, V. Giordanengo and J.C. Lefebvre, High permissivity of human HepG2 hepatoma cells for influenza viruses, J Clin Microbiol 42 (2004), pp. 5861-5865. 
Vaccine Volume 24, Issues 44-46, 10 November 2006, Pages 6717-6723

Proceedings of the Second European Influenza Conference, Proceedings of the Second European Influenza Conference

[11] M. Zambon, Cell culture for surveillance on influenza, Dev Biol Stand 98 (1999), pp. 65-71 discussion 73-4.

[12] M. Zambon, Laboratory containment for influenza A H5N1 virus: level 2, level 3, or level 3+?, Commun Dis Public Health 1 (1998), pp. 71-72.

[13] I. Stephenson, J.M. Wood, K.G. Nicholson and M.C. Zambon, Sialic acid receptor specificity on erythrocytes affects detection of antibody to avian influenza haemagglutinin, J Med Virol 70 (2003), pp. 391-398.

[14] A. Meijer, A. Bosman, E.E.H.M. van de Kamp, B. Wilbrink, M. Du Ry van Beest Holle and M. Koopmans, Measurement of antibodies to avian influenza virus $A(H 7 N 7)$ in humans by hemagglutination inhibition test, J Virol Methods 132 (2006), pp. 113-120.

[15] Y. Thomas, L. Kaiser and Wunderli W, EISS Task Group on Near Patient Test. The use of near patient tests in influenza surveillance: Swiss experience and EISS recommendations, Euro Surveill 8 (2003), pp. 240-246.

[16] T. Rowe, R.A. Abernathy, J. Hu-Primmer, W.W. Thompson, X. Lu and W. Lim et al., Detection of antibody to avian influenza A (H5N1) virus in human serum by using a combination of serologic assays, J Clin Microbiol 37 (1999), pp. 937-943.

[17] M. Kiso, K. Mitamura, Y. Sakai-Tagawa, K. Shiraishi, C. Kawakami and K. Kimura et al., Resistant influenza A viruses in children treated with oseltamivir: descriptive study, Lancet 364 (2004), pp. 759-765.

[18] Anonymous, NISN statement on antiviral resistance in influenza viruses, Wkly Epidemiol Rec 79 (2004), pp. 306-308. 